С. Г. СолодкиЙ (Ии-гт математики НАН Ухраины, Кнев)

\title{
ОПТИМИЗАЦИЯ ПРОЕКЦИОННЫХ СХЕМ ДИСКРЕТИЗАЦИИ НЕКОРРЕКТНЫХ ЗАДАЧ
}

New projection schemes of digitization of ill-posed problems are constructed which are optimal in the sense of amount of used discrete information. The fact is established that the application of self-adjoint projection schemes to digitization of equations with self-adjoint operators is not optimal.

Побуловапо пові проехційıі схеми цискретизапій пекорек пиих зајач, що є оптимазьыими у сепсі

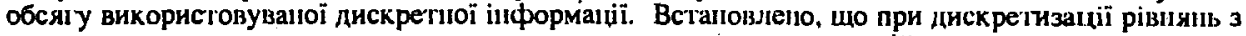
самоспряженими операторами використания самоспряжених проекційих схем не є оптимальним.

В настоящее время большой интерес вызывают исследования по оценкам информационной сложности приближенного решения различных задач (см. [1]). При этом под сложностью понимается минимальное количество значений информационных функционалов, требуемых для нахождения решения задачи с наперед заданной точностью. Применительно к операторным уравнениям подобные исследования проводились в основном для уравнений II рода (см., например, [2] и приведенную в ней библиографию), уравнения I рода изучались в работах [3-7]. Целью настоящей статьи является построение новых проекционных схем дискретизации некорректных задач, оптимальных (как по точности приближения, так и в смысле объема используемой дискретной информации) на ряде классов уравнений I рода. В частности, будет установлен неожиданный на наш вэгляд эффект, состоящий в том, что при дискретизации уравнений с самосопряженными операторами использование самосопряженных приближающих операторов не является, вообще говоря, оптимальным в указанном смысле.

Пусть $X$ - действительное гильбертово пространство с обычным скалярным произведением $(\cdot, \cdot)$ и порождаемой им нормой $\|\cdot\|_{X}$, a $\mathcal{L}(X, X)$ - пространство линећных непрерывных операторов из $X$ в $X$ с введенной в нем стандартной нормой $\|\cdot\|$. Для уравнения I рода

$$
A x=f
$$

с компактным оператором $A \in \mathcal{L}(X, X)$ и $f \in \operatorname{Range} A$ рассмотрим задачу конечномерной аптроксимации нормального решения $x_{0}$, т. е. решения уравнения (1) с минимальной нормой в $X$. Будем считать при этом, что вместо $f$ задано некоторое его приближение $f_{\delta} \in X_{\delta, f}$, где $X_{\delta, f}$ - шар с центром в $f$ радиуса $\delta$ в метрике пространства $X$, а $\delta$ - малое положительное число, называемое модельной погрешностью правои части (1). Другими словами, мы имеем возмущенное уравненне

$$
A x=f_{\delta},
$$

где $\left\|f-f_{\delta}\right\|_{X} \leq \delta$.

Поскольку при организащии любой вычислительной процедуры допустимо использование лишь конечного числа значений информационных функционалов, вычисленных на коэффициентах задачи, то вместо уравнения (2) мы вынуждены рассматривать его конечномерның аналог. Этот этап построения приближенного решения (1), (2) в теории некорректных задач принято называть (см., например, [8]) дискретизацией, суть которой заключается в том, что в процессе вычислекня используется только дискретная информация об операторе $A$ и правой части $f_{\delta}$. В рамках настоящей работы ограничимся изучением проекционных схем дискретизащии. В следующем пункте приведем некоторые изве- 
стные понятия и факты, а также дадим постановку исследуемой задачи.

1. Пусть $B=\left\{b_{1}, b_{2}, \ldots, b_{m}, \ldots\right\}$ - произвольның ортонормированнын базис гильбертова пространства $X$, а $P_{m}=P_{B, m}$ - ортопроектор на $\operatorname{span}\left\{b_{1}\right.$, $\left.b_{2}, \ldots, b_{m}\right\}$, т. е. $P_{B, m} g=\sum_{i=1}^{m}\left(b_{j}, g\right) b_{i}$. Тогда произвольный оператор $A \in$ $\in \mathcal{L}(X, X)$ может быть представлен с помошью бесконечной матрицы $\left\{\left(b_{i}, A b_{j}\right)\right\}_{i, j=1}^{\infty}$ в следующем виде:

$$
A g=\sum_{i, j=1}^{\infty}\left(b_{i}, A b_{j}\right)\left(b_{j}, g\right) b_{i} .
$$

Каждому скалярному произведению $\left(b_{i}, A b_{j}\right)$ поставим в соответствие точку $(i, j)$ из множества $[1, \infty] \times[1, \infty]$ координатной плоскости, которую будем рассматривать в качестве номера функционала $\left(b_{i}, A b_{j}\right)$. Номером скалярного произведения $\left(b_{i}, g\right)$ будем считать число $j$. Если теперь каждой области. $\Omega \subset$ $\subset[1, \infty) \times[1, \infty)$ поставнть в соответствие выражения

$$
\begin{gathered}
A_{\Omega}=A_{B, \Omega}:=\sum_{(i, j) \in \Omega}\left(b_{i}, A b_{j}\right)\left(b_{j}, \cdot\right) b_{i}, \\
P_{\Omega} f_{\delta}=\sum_{k \in \omega}\left(b_{k}, f_{\delta}\right) b_{k}, \quad \omega=\{i:(i, j) \in \Omega\},
\end{gathered}
$$

то с помощью различных наборов $\Omega$ и $B$ можно определить все возможные проекционные схемы дискретизации уравнений (1), (2), в которых в качестве дискретной информации используются скалярные произведения

$$
\left(b_{i}, A b_{j}\right), \quad\left(b_{i}, f_{\delta}\right), \quad(i, j) \in \Omega, \quad k \in \omega,
$$

называемые также галеркинской информацией. Под проекционнон схемой дискретизации (1), (2) в дальнейшем будем понимать пару $(\Omega, B)$ элементов $\Omega$ и $B$, в результате воздеиствия которой на (2) осуществляется переход к дискретизированному уравнению

$$
A_{\Omega} x=P_{\Omega} f_{\delta},
$$

где $A_{\Omega}$ и $P_{\Omega} f_{\delta}$ определяются согласно (3).

Поскольку в силу компактности оператора $A$ задача нахождения решений уравнения (1) не является корректной в смысле Адамара, то для построения устоичивого приближенного решения (1) необходима регуляризация: Следуя $[9$, c. $55 ; 10$, с. 7$]$, под регуляризатором задачи $(1)$, (2) будем понимать такое семейтво операторов $R_{\alpha}=R_{\alpha}(A): X \rightarrow X$, зависяших от параметра $\alpha=\alpha(\delta)$ и оператора $A$, что для любого $f \in \operatorname{Range} A$

$$
\lim _{\delta \rightarrow \infty} \sup _{f_{\delta} \in X_{\delta, f}} \inf _{u \in A^{-1} f}\left\|u-R_{\alpha}(A) f_{\delta}\right\|_{X}=0,
$$

где $a(\delta) \rightarrow 0$ при $\delta \rightarrow 0$, а $A^{-1} f$ - полный прообраз элемента $f$. Совокупность всех регуляризаторов обозначим через $R$.

Определение. Под регуляризационно-проекционныл методом решения уравнения (1) будель понихьть произвольное правило $\left(R_{\alpha}, \Omega, B\right), R_{\alpha} \in \mathcal{R}$, ө соответстөии с которылі набору функиионалов (4) в качестве приближенного решения (1) сопоставлгется элехент

$$
x_{\text {disc }}=x_{\text {disc }}\left(R_{\alpha}, \Omega, B, A, f_{\delta}\right):=R_{\alpha}\left(A_{B, \Omega}\right) P_{\Omega} f_{\delta} .
$$

Таким образом, регуляризащионно-проекционнын метод (РПМ) решения (1) 
можно представить в виде комбинаџии метода регуляризации $R_{\alpha}$ и проекционнон̆ схемы дискретизашии $(\Omega, B)$.

Предположим, что нормаљьое решение $x_{0}$ уравнения (1) принадлежит некоторому ограннченному центрально-симметричному множеству $\mathcal{M} \subset X$. Точность PIM $\left(R_{\alpha}, \Omega, B\right)$ на множестве $\mathcal{M}$ характернзуется наиболышим отклонением

$$
\mathscr{E}_{\delta}\left(A, \mathcal{M}, R_{\alpha}, \Omega, B\right)=\sup _{x_{0} \in \mathcal{M} f_{\delta}:\left\|A x_{0}-f_{\delta}\right\|_{x} \leq \delta}\left\|x_{0}-x_{\text {disc }}\right\|_{X} .
$$

Элементы множества $M_{p, p}(A):=\left\{u: u=|A|^{p} v, v \in X_{\rho, 0}\right\},|A|=\left(A^{*} A\right)^{1 / 2}$, где $A^{*}$ - оператор, сопряженный $\mathrm{x} A$, называются истокопредставимыми. Известно, что если уравнение (1) имеет истокопредставимое решение $x_{0} \in$ $\in M_{p, p}(A)$, то $x_{0}$ - наименьшее в метрике $X$ решение (1) (т. е. $x_{0}$ - нормальное решение (1)). В дальнеишем будем счнтать, что при некоторых $p>0$ и $\rho>$ $>0$ уравнение (1) имеет решение $x_{0} \in M_{p, p}(A)$. Если теперь ввести в рассмотрение некоторый класс компактных операторов $\mathcal{H} \subset\{A: A \in \mathcal{L}(X, X),\|A\| \leq$ $\leq \gamma\}$, то под погрешностью PIM $\left(R_{\alpha}, \Omega, B\right)$ на классе $\mathcal{H}$, как обычно, будем понимать величину

$$
\mathscr{E}_{\delta, p, p}\left(\mathcal{H}, R_{\alpha}, \Omega, B\right)=\sup _{A \in \mathscr{H}} \mathscr{E}_{\delta}\left(A, M_{p, p}(A), R_{\alpha}, \Omega, B\right) .
$$

Оптимальной погрешностью проекционной схемы $(\Omega, B)$ на классе $\mathcal{H}$ назовем величину.

$$
\mathscr{E}_{\delta, p, p}(\mathcal{H}, \Omega, B)=\sup _{R_{\alpha} \in R} \mathscr{E}_{\delta, p, p}\left(\mathcal{H}, R_{\alpha}, \Omega, B\right) .
$$

Известно, что при любых $\Omega$ и $B$ справедливо соотношение

$$
\mathscr{E}_{\delta, p, \rho}(\mathcal{H}, \Omega, B) \geq \rho^{1 /(p+1)} \delta^{p /(p+1)} .
$$

Прежде чем ввести в рассмотрение исследуемую величину, установим следующее утверждение.

Теорема 1. Дия сохранения оптильального порядка точности $O\left(\delta^{p /(p+1)}\right)$ на классе компактных операторов $\mathcal{H}$ необходимь дискретизировать уравнение (2) так, чтобы для любого оператора $A \in \mathcal{H}$ и элемента $x_{0} \in M_{p, p}(A)$ выполналось соотночение

$$
\left\|\left(P_{\Omega} A-A_{\Omega}\right) x_{0}\right\|_{X}=O(\delta) .
$$

Доказательстөо. Пусть при некоторых $A$ и $\bar{x}_{0}=|A|^{p} \bar{v},\|\bar{v}\|=\rho$, справедливо равенство

$$
\left\|\left(P_{\Omega} A-A_{\Omega}\right) \bar{x}_{0}\right\|_{X}=\delta_{1} .
$$

Тогда (5) можно рассматривать в качестве возмушенного уравнения по отношению $\mathrm{k}$ уравнению

$$
A_{\Omega} x=g,
$$

где $g=A_{\Omega} \bar{x}_{0}$, с правой частью $P_{\Omega} f_{\delta}$, известной с некоторой погрешностью $\delta_{2}$. Оценим величину $\delta_{2}$. Используя (8), находим

$$
\left\|g-P_{\Omega} f_{\delta}\right\|_{X} \leq\left\|P_{\Omega}\left(\bar{f}-f_{\delta}\right)\right\|_{X}+\left\|\left(P_{\Omega} A-A_{\Omega}\right) \bar{x}_{0}\right\|_{X} \leq \delta+\delta_{1}
$$


где $\bar{f}=A \bar{x}_{0}$. С другони стороны, для любого $f_{\delta}$ такого, что $P_{\Omega} f_{\delta}=R_{\Omega} \bar{f}$ и $\left\|\left(I-P_{\Omega}\right)\left(\bar{f}-f_{\delta}\right)\right\| \leq \delta$, в силу (8) справедливо $\left\|g-P_{\Omega} f_{\delta}\right\|_{X}=\delta_{1}$. Таким образом, имеем $\delta_{1} \leq \delta_{2} \leq \delta+\delta_{1}$.

Для любого центрально-симметричного множества $\mathcal{M}$ и произвольного регуляризатора $R_{\alpha} \in R$ введем в рассмотрение величнну

$$
e_{\delta, \delta_{2}}\left(A, A_{\Omega}, \mathcal{M}, R_{\alpha}\right)=\sup _{x_{0} \in \mathcal{M} f_{\delta}:\left\|A x_{0}-f_{\delta}\right\|_{X} \leq \delta}\left\|x_{0}-x_{\text {disc }}\right\|_{X},
$$

где $x_{\text {disc }}$ определяется согласно (6). Используя рассуждения из § 4.2 [8], § 1.3 [10], легко показать, что

$$
e_{\delta . \delta_{2}}\left(A, A_{\Omega}, \mathcal{M}, R_{\alpha}\right) \geq \bar{\omega}_{\delta_{2}}\left(A_{\Omega}, \mathcal{M}\right),
$$

где $\bar{\omega}_{\delta}(F, \mathcal{M})=\sup _{u \in \mathcal{M},\left\|F_{u}\right\|_{x} \leq \delta}\|u\|_{X}$. Нетрудно видеть, что для любого $\xi \geq 1$

$$
\bar{\omega}_{\delta_{2}}\left(A_{\Omega}, M_{p, \rho}(A)\right)=\frac{1}{\xi} \bar{\omega}_{\xi \delta_{2}}\left(A_{\Omega}, M_{p, \xi \rho}(A)\right) \geq \frac{1}{\xi} \bar{\omega}_{\xi \delta_{2}}\left(A_{\Omega}, M_{p, \rho}(A)\right) .
$$

В силу компактности $A$ при достаточно мальх $\delta, \delta$, число $\lambda=$ $=\left(\delta_{2} / \rho\right)^{1 /(p+1)}$ будет принадлежать спектру оператора $|A|$. Если $\bar{v}-$ собственный элемент оператора $|A|(|A| \bar{v}=\lambda \bar{v})$, то из (8) и $\|A y\|_{X}=\|A \mid y\|_{X}$ следует

$$
\begin{gathered}
\left\|A_{\Omega}|A|^{p} \bar{v}\right\|_{X} \leq \\
\leq\left\|\left(P_{\Omega} A-A_{\Omega}\right)|A|^{p} \bar{v}\right\|_{X}+\left\|P_{\Omega} A|A|^{p} \bar{v}\right\|_{X} \leq \delta_{1}+\delta_{2} \leq \xi \delta_{2}, \\
\left\||A|^{p} \bar{v}\right\|_{X} \leq \rho^{1 /(p+1)} \delta_{2}^{p /(p+1)},
\end{gathered}
$$

где $\xi=1+\delta_{1} / \delta_{2}$ и $1<\xi \leq 2$.

Отсюда вытекает оценка

$$
e_{\delta, \delta_{2}}\left(A, A_{\Omega}, M_{p, p}(A), R_{\alpha}\right) \geq \frac{1}{\xi} \bar{\omega}_{\xi \delta_{2}}\left(A_{\Omega}, M_{p, p}(A)\right) \geq \frac{1}{\xi} \rho^{1 /(p+1)} \delta_{2}^{p /(p+1)} .
$$

Утверждение теоремы следует из очевидного соотношения

$$
\mathscr{E}_{\delta}\left(A, M_{p, \rho}(A), R_{\alpha}, \Omega, B\right) \geq e_{\delta, \delta_{2}}\left(A, A_{\Omega}, M_{p, p}(A), R_{\alpha}\right),
$$

оценки $\delta_{2} \geq \delta_{1}$ и того факта, что область $\Omega$ строится независимо от оператора $A$ и элемента $\bar{v}$.

Замечание 1. Ранее в работе [11], где изучалась конечно-разностная схема дискретизащии, рассматривался следующий аналог условия (7):

$$
\left\|\left(P_{\Omega} A-A_{\Omega}\right) x_{0}\right\|_{X} \rightarrow 0 \text { при } \delta \rightarrow 0 .
$$

Через $\Pi_{\delta, p, p}^{d}(\mathcal{H}), d=\left(d_{1}, d_{2}\right)$, обозначим множество всех возможных проекционных схем $(\Omega, B)$, для которых выпоняются соотншения

$$
\mathscr{E}_{\delta, p, p}(\mathcal{H}, \Omega, B) \leq d_{1} \rho^{1 /(p+1)} \delta^{p /(p+1)}, \quad d_{1} \geq 1,
$$

и для люобого уравнення (1), где $A \in \mathcal{H}, f=A x_{0}, x_{0} \in M_{p, p}(A)$, 


$$
\left\|\left(P_{\Omega} A-A_{\Omega}\right) x_{0}\right\|_{X} \leq d_{2} \delta .
$$

Предполагается, что постоянные $d_{1}, d_{2}$ выбраны так, что $\Pi_{\delta, p, p}^{d}(\mathcal{H}) \neq \varnothing$. Это будет выполняться, например, если

$$
\left(d_{2}+1\right)^{2 p+1} \leq\left(d_{1}\left(2 d_{2}+1\right)\right)^{p+1} .
$$

Пусть $\operatorname{card}(\Omega)$ - общее число функционалов (4), используемых при дискретизации (5) уравнения (2). Настоящая статья посвящена вычислению величины

$$
\operatorname{Card}_{\delta, p, \rho}(\mathcal{H})=\min \left\{\operatorname{card}(\Omega):(\Omega, B) \in \Pi_{\delta, p, \rho}^{d}(\mathcal{H})\right\},
$$

которая характеризует минимальный объем дискретной информации (4), гарантирующий оптимальный порядок точности $O\left(\delta^{p /(p+1)}\right)$ на классе уравнений (1) с операторами $A \in \mathcal{H}$ и нормальными решениями $x_{0}$ из множества $M_{p, p}(A)$.

2. Обозначим через $X^{r}, 0<r<\infty$, линейное нормированное подпространство $X$ такое, что для произвольного элемента $g \in X^{r}$ справедливо соотношение $\|g\|_{X} \leq\|g\|_{X^{r}}$ и при этом найдется такой базис $B$, что для любого $k=$ $=1,2, \ldots$ выполняются двойственные соотношения вида

$$
\left\|I-P_{B, k}\right\|_{X^{r} \rightarrow X} \leq \beta_{r} k^{-r}, \quad\left\|P_{B, k}\right\|_{X \rightarrow X^{r}} \leq \bar{\beta}_{r} k^{r},
$$

где $I$ - тождественный оператор в $X$, а константы $\beta_{r}$ н. $\bar{\beta}_{r}$ не зависят от $k$.

Совокупность базисов $B$, удовлетворяющих этим условиям, обозначим через $\mathcal{B}_{r}$. В дальнейшем ограничимся рассмотрением базисов только из $\mathcal{B}_{r}$.

- Пусть $\mathcal{H}_{\gamma}^{r}=\left\{A: A \in \mathcal{L}(X, X),\|A\|_{X \rightarrow X^{r}} \leq \gamma,\left\|A^{*}\right\|_{X \rightarrow X^{r}} \leq \gamma\right\}$. Легко видеть, что $X^{r}$ является обобщением соболевского пространства $W_{2}^{r}$ функций, имеющих суммируемые в квадрате $r$-е пронзводные, а множество $\mathscr{H}_{\gamma}^{r}$ обобщает класс интегральных операторов вида $A x(t)=\int_{0}^{1} h(t, \tau) x(\tau) d \tau$, ядра $h(t, \tau)$ которых имеют частные производные и

$$
\sum_{0 \leq i+j \leq r}\left(\int_{0}^{l} \int_{0}^{l}\left|\frac{\partial^{i+j} h(t, \tau)}{\partial t^{i} \partial \tau^{j}}\right|^{2} d t d \tau\right)^{l / 2} \leq \gamma .
$$

Для $X^{r}=W_{2}^{r}$ в качестве примера базиса из $\mathcal{B}_{r}$ можно назвать тригонометрическую систему (в периодическом случае) или ортонормированную систему функция, построенных на базе так называемых всплесков (подробнее об этом cM. [12]).

В рамках предлагаемой нами проекционной схемы дискретизации под $\Omega$ будем понимать фигуру координатной плоскости следующего вида:

$$
\begin{gathered}
\Gamma_{n}^{a, b}=\bigcup_{k=0}^{n} Q_{k}, \\
Q_{0}=\{1\} \times\left[1,2^{b n}\right], \quad Q_{k}=\left(2^{k-1}, 2^{k}\right] \times\left[1,2^{b n-a k}\right], \quad k=1,2, \ldots, n,
\end{gathered}
$$

где $b \geq a \geq 0$. Заметим, что коордннаты $(i, j)$ верхних правых углов прямоугољников $Q_{k}$ лежат на гиперболе $i^{a} j=2^{b n}$, а сама фигура $\Gamma_{n}^{a, b}$ представляет собой квадрант так называемого ступенчатого гитерболического креста. Впервые гиперболическии крест был применен в теории приближений К. Н. Бабенко в работе [13], где для некоторых классов периодических функщий иссле- 
довался вопрос об оптимальном приближающем подпространстве в смысле поперечника Колмогорова. При дискретизации нехоррехтных задач квадрант ступенчатого гиперболического креста $\Gamma_{n}^{a, b}, a=1, b=2$, уже использовался в работе [5]. При этом у ядер соответствующих интегральных операторов предполагалось наличие суммируемой в квадрате доминирующей смешанной частной производной. Мы же сећчас покажем, что на самом деле подход, связанный с использованием гиперболического хреста, может быть успешно применен и для значительно более широких классов уравнений (1), где $A \in \mathcal{H}_{\gamma}^{r}$.

Следуя [14], рассмотрим класс регуляризаторов, задаваемых параметрически. А именно, пусть $\mathcal{G}=\left\{g_{\alpha}\right\}, 0<\alpha<1$, - некоторое параметрическое семейство функций, измеримых по Борелю на отрезке $\left[0, \gamma^{2}\right]$, $\|A\| \leq \gamma$, и при $0 \leq p \leq p_{*}$, удовлетворяющих условиям

$$
\sup _{0 \leq \lambda \leq \gamma^{2}} \lambda^{p}\left|1-\lambda g_{\alpha}(\lambda)\right| \leq \chi_{p} \alpha^{p}, \quad \sup _{0 \leq \lambda \leq \gamma^{2}} \lambda^{1 / 2}\left|g_{\alpha}(\lambda)\right| \leq \chi_{*} \alpha^{-1 / 2},
$$

где $p_{*}, \chi_{p}$ и $\chi_{*}$ - некоторые не зависящие от $\alpha$ положительные константы.

Совокупность регуляризаторов, задаваемых соотношением $R_{\alpha}=$ $=g_{\alpha}\left(A^{*} A\right) A^{*}, g_{\alpha} \in G$, обозначим $R^{\prime} \subset R$. Примером регуляризатора из $R^{\prime}$ является обобщенный метод Тихонова [4].

Согласно схеме $\left(\Gamma_{n}^{a, b}, B\right)$, каждому оператору $A \in \mathcal{H}_{\gamma}^{r}$ ставится в соответствие оператор

$$
A_{n}=A_{a, b, n}:=\sum_{k=0}^{n} \Delta_{k} A P_{2^{b n-a k}},
$$

где $\Delta_{0}=P_{1}, \Delta_{k}=P_{2^{k}}-P_{2^{k-1}}, k=1,2, \ldots, n$, a $P_{n}=P_{B, n}$ - opтопроектор, действующий на линейную оболочку первых $n$ элементов базиса $B \in \mathcal{B}_{r}$. Если $b n-a k$ не является целым числом, то под $P_{2 b n-a k}$ будем понимать $P_{2\{b n-a k\}}$, где $\{g\}-$ - лижайшее сверху к $g$ целое число. В качестве приближенного решения $x_{\text {disc }}(6)$ будем рассматривать элемент

$$
x_{\mathrm{disc}}=g_{\alpha}\left(A_{a, b, n}^{*} A_{a, b, n}\right) A_{a, b, n}^{*} P_{2^{n}} f_{\delta},
$$

где $g_{\alpha}$ 一 произвольная функция из $\mathcal{G}, p_{*} \geq p / 2$.

Следующий результат доказывается аналогично теореме 2 из [6].

Теорема 2. Пусть $\alpha \approx \delta^{2 /(p+1)}$ и $B-$ произвольный базис из $\mathcal{B}_{r}$. Для того чтобы $\left(\Gamma_{n}^{a, b}, B\right) \in \Pi_{\delta, p, p}^{d}\left(\mathcal{H}_{\gamma}^{r}\right)$, достаточно выполнения условий:

a) $2^{-r(p+1) n} \times \delta, \quad a=0, \quad b=1 \quad$ npu $\quad 0<p \leq 1$;

б) $2^{-r(p+1) n} \times \delta, \quad a=\frac{3-p}{2}, \quad b=2$ npu $1<p<2$;

в) $2^{-2 r(p+1) n / p}=\delta, \quad a=\frac{p-1}{p}, \quad b=2$ npu $p \geq 2$.

Оптильальный порядок точности $О\left(\delta^{p /(p+1)}\right)$ достигается при использовании произвольного регуялризатора $R_{\alpha} \in R^{\prime}$. При этом

$$
\operatorname{Card}_{\delta, p, \rho}\left(\mathcal{H}_{\gamma}^{r}\right) \leq \operatorname{card}\left(\Gamma_{n}^{u, b}\right) \times \delta^{-y}
$$


где $y=\frac{2}{r(p+1)}$ npu $0<p \leq 1, y=\frac{p+3}{2 r(p+1)}$ npu $1 \leq p \leq 2, y=\frac{2 p+1}{2 r(p+1)}$ npu $p \geq 2$.

Заметим, что утверждение теоремы 2 при $0<p \leq 1$ следует из результатов работы [4].

3. Рассмотрим теперь случай, когда оператор $A$ в (1) является самосопряженным и неотрицательным. Положим

$$
\begin{gathered}
\hat{\mathcal{H}}_{\gamma}=\left\{A: A \in \mathcal{L}(X, X),\|A\| \leq \gamma, A=A^{*} \geq 0\right\}, \\
\hat{\mathcal{H}}_{\gamma}^{r}=\hat{\mathcal{H}}_{\gamma} \cap \mathcal{H}_{\gamma}^{r} .
\end{gathered}
$$

Поскольку при решении задачи (1), где $A=A^{*} \geq 0$, аппроксимируюший оператор $A_{\Omega}$ принято строить (см., например, [10, с. 31]) также самосопряженным (необязательно неотрицательным), то ограничимся ниже множеством $\hat{\Pi}_{\delta, p, p}^{d}\left(\hat{\mathcal{H}}_{\gamma}^{r}\right) \subset \Pi_{\delta, p, \rho}^{d}\left(\hat{\mathcal{H}}_{\gamma}^{r}\right)$ проекционных схем с симметричными относительно биссектрисы областями $\Omega$ координатной плоскости. При этом эффективность дискретизации уравнений (1), (2) в рамках РПМ будем исследовать с помощью величины

$$
\widehat{\operatorname{Card}}_{\delta, p, \rho}\left(\hat{\mathcal{H}}_{\gamma}^{r}\right)=\min \left\{\operatorname{card}(\Omega):(\Omega, B) \in \hat{\Pi}_{\delta, p, \rho}^{d}\left(\hat{\mathcal{H}}_{\gamma}^{r}\right)\right\} .
$$

Класс регуляризаторов, задаваемых функцией от оператора решаемого уравнения, определим следующим образом. Пусть $\hat{G}=\left\{g_{\alpha}\right\}, 0<\alpha<1$, - некоторое параметрическое семейство функций, измеримых по Борелю на отрезке $\left[-\gamma_{0} \alpha, c_{*} \gamma\right], \gamma_{0}>0, c_{*} \geq 1 \quad\left(\gamma_{0}\right.$, вообще говоря, зависит от конкретной функции $g_{\alpha}$ ) и при $0 \leq p \leq \hat{p}_{*}$ удовлетворяющих следующим условиям:

$$
\begin{gathered}
\sup _{-\gamma_{0} \alpha \leq \lambda \leq c_{*} \gamma}|\lambda|^{p}\left|1-\lambda g_{\alpha}(\lambda)\right| \leq \hat{\chi}_{\alpha} \alpha^{p}, \\
\sup _{-\gamma_{0} \alpha \leq \lambda \leq c_{*} \gamma}\left|g_{\alpha}(\lambda)\right| \leq \hat{\chi}_{*} \alpha^{-1},
\end{gathered}
$$

где $\hat{p}_{*}, \hat{\chi}_{p}$ и $\hat{\chi}_{*}$ - как и прежде, некоторые не зависящие от $\alpha$ положительные константы. Совокупность регуляризаторов вида $R_{\alpha}=g_{\alpha}(A), g_{\alpha} \in \hat{G}$. обозначим через $\hat{R}^{\prime}$.

Пример. Пусть $q=0,1,2, \ldots$. Согласно обобшенному методу Лаврентьева [3] уравнениям (1), (2) ставится в соответствие регуляризованное уравнение $\left(\alpha^{q+1} I+A^{q+1}\right) x=A^{q} f_{\delta}$. Этот метод регуляризации из $\hat{R}^{\prime}$. с функцией $g_{\alpha}(\lambda)=\frac{\lambda^{q}}{\alpha^{q+1}+\lambda^{q+1}}$, для которои выполнены условия (10) при $\hat{p}_{*}=q+1$, $c_{*}=1$ и любых $0<\gamma_{0}<1, \gamma>0$. В случае $q=0$ получаем обычный метод Лаврентьева. В качестве других примеров регуляризаторов из $\hat{R}^{\prime}$ можно назвать известные итеращионные процедуры Ландвебера, Факеева - Ларди и др. (подробнее см. $[3,10]$ ).

Аналогично теореме 1 [7] устанавливается следуюший результат.

Tеорема 3. Пусть $\alpha=\mu_{1} \delta^{1 /(p+1)},\left\|A-A_{\Omega}\right\| \leq \gamma_{0} \alpha$ и B- произвольный базис из $\mathcal{B}_{r}$. Тогда на классе уравнений(1) с операторахц $A \in \hat{\mathcal{H}}_{\gamma} u x_{0} \in$ $\in M_{p, \rho}(A)$ для любой проекционной схелєы $(\Omega, B)$ и произвольного регуляриза- 
тора $R_{\alpha}$ из $\hat{R}^{\prime}, \hat{p}_{*} \geq p$, справедлива оценка

$$
\begin{aligned}
\left\|x_{0}-x_{\text {disc }}\right\|_{X} \leq & \left(\hat{\chi}_{*} \mu_{1}^{-1}+\rho \hat{\chi}_{p} \mu_{1}^{p}\right) \delta^{p /(p+1)}+\rho \hat{\chi}_{0}\left\|A^{p}-\left|A_{\Omega}\right|^{p}\right\|+ \\
& +\hat{\chi}_{*} \mu_{1}^{-1} \delta^{-1 /(p+1)}\left\|\left(A-A_{\Omega}\right) x_{0}\right\|_{X} .
\end{aligned}
$$

В качестве $\Omega$ возьмем ступенчатый гиперболический крест вида

$$
\hat{\Gamma}_{n}=\{1\} \times\left[1,2^{2 n}\right] \bigcup_{k=1}^{2 n}\left(2^{k-1}, 2^{k}\right] \times\left[1,2^{2 n-k}\right] .
$$

Тогда каждому оператору $A \in \hat{\mathcal{H}}_{\gamma}^{r}$ будет соответствовать конечномерный оператор $A_{\Omega}=A_{n}:=\sum_{k=0}^{2 n} \Delta_{k} A P_{2^{2 n-k}}$, где, как и ранее, $P_{n}=P_{B, n}$ - ортопроектор на первые $n$ элементов базиса $B \in \mathcal{B}_{r}$. Приближенное решение $x_{\text {disc }}$ будет задаваться соотношением $x_{\text {disc }}=g_{\alpha}\left(A_{n}\right) P_{22 n} f_{\delta}$, где $g_{\alpha}$ - произвольная функция из $\hat{\mathcal{G}}, \hat{p}_{*} \geq p$.

Приведем теперь ряд вспомогательных результатов, которые потребуются в дальнейшем. Пусть $A, H \in \hat{\mathcal{H}}_{\gamma}^{r}$. Для любого $p>0$ справедлива оценка [4]

$$
\left\|A^{p}-H^{p}\right\| \leq a_{p}\|A-H\|^{\min \{p, 1\}},
$$

где $a_{p}=\frac{4}{\pi}$ при $p \leq 1$ и функция $p \rightarrow a_{p}$ ограничена на $\left(0, \hat{p}_{*}\right]$ для всех $\hat{p}_{*}>0$.

Лемма 1. Пусть $A \in \hat{\mathcal{H}}_{\gamma}^{r}$ и $p>1$. Тогда прu $n \geq 3$

$$
\begin{gathered}
\left\|\left(A-A_{n}\right) A^{p}\right\| \leq v_{p} 2^{-2 r n} \sqrt{n}, \\
\left\|A^{2}-A_{n}^{2}\right\| \leq v_{*} 2^{-2 r n} n, \\
\left\|\left(A-A_{n}\right)\right\| \leq 2 \gamma \beta_{r} 2^{-m},
\end{gathered}
$$

гдe $v_{*}=(1+2 \sqrt{2}) 2^{r} \gamma^{2} \beta_{r}^{2}, v_{p}=\left(2+2^{r}\right) \gamma^{p+1} \beta_{r}^{2}$.

Доказательство. Прежде всего запишем разложение

$$
A-A_{n}=\left(I-P_{2^{2 n}}\right) A+\sum_{k=0}^{2 n} \Delta_{k} A\left(I-P_{2^{2 n-k}}\right) .
$$

С учетом определений $\hat{\mathcal{H}}_{\gamma}^{r}$ и $X^{r}$ для любого $p>1$ имеем

$$
\begin{gathered}
\left\|\left(I-P_{2^{2 n}}\right) A^{p+1}\right\| \leq \gamma^{p+1} \beta_{r}^{2} 2^{-2 m}, \\
\left\|\sum_{k=0}^{n} \Delta_{k} A\left(I-P_{2^{2 n-k}}\right) A^{p}\right\| \leq \sum_{k=0}^{n}\left\|\left(I-P_{2^{2 n-k}}\right) A\right\|^{2}\|A\|^{p-1} \leq \\
\leq \frac{2^{r}}{2^{r}-1} \gamma^{p+1} \beta_{r}^{2} 2^{-2 m}, \\
\left\|\sum_{k=n+1}^{2 n} \Delta_{k} A\left(I-P_{2^{2 n-k}}\right) A^{p}\right\| \leq \sup _{\|g\|_{X} \leq 1}\left\|\sum_{k=n+1}^{2 n} A^{p}\left(I-P_{2^{2 n-k}}\right) A \Delta_{k} g\right\|_{X} \leq
\end{gathered}
$$




$$
\begin{gathered}
\leq\|A\|^{p-1} \sup _{\|g\|_{x} \leq 1}\left(\sum_{k=n+1}^{2 n}\left\|\Delta_{k} g\right\|_{X}^{2}\right)^{1 / 2}\left(\sum_{k=n+1}^{2 n}\left\|\Delta_{k} A\right\|^{2}\left\|\left(I-P_{2^{2 n-k}}\right) A\right\|^{2}\right)^{1 / 2} \leq \\
\leq 2^{r} \gamma^{p+1} \beta_{r}^{2} 2^{-2 m} \sqrt{n} .
\end{gathered}
$$

Объединяя полученные оценки, получаем (14).

Для нахождения оценки (15) воспользуемся представлением $A_{n}^{2}=$ $=\sum_{k=0}^{2 n} P_{2^{2 n-k}} A \Delta_{k} A P_{2^{2 n-k}}$. Тогда имеем

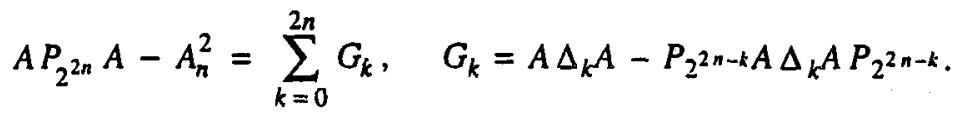

Заметкм далее, что для любого $g \in X_{1,0}\left(\|g\|_{X} \leq 1\right)$ выполняется

$$
\begin{gathered}
\left\|G_{k} g\right\|_{X} \leq\left\|A \Delta_{k} A\left(I-P_{2^{2 n-k}}\right) g\right\|_{X}+ \\
+\left\|\left(I-P_{2^{2 n-k}}\right) A \Delta_{k} A P_{2^{2 n-k}} g\right\|_{X} \leq \\
\leq\left\|\Delta_{k} A\right\|\left\|\left(I-P_{2^{2 n-k}}\right) A\right\|\left(\left\|P_{2^{2 n-k}} g\right\|_{X}+\left\|\left(I-P_{2^{2 n-k}}\right) g\right\|_{X}\right),
\end{gathered}
$$

откуда непосредственно следует

$$
\left\|A P_{2^{2 n}} A-A_{n}^{2}\right\| \leq \sup _{\|g\|_{X} \leq 1} \sum_{k=0}^{2 n}\left\|G_{k} g\right\|_{X} \leq \sqrt{2} 2^{r} \gamma^{2} \beta_{r}^{2} 2^{-2 m}(2 n+1) .
$$

Подставляя найденкую оценку в соотношение

$$
\left\|A^{2}-A_{n}^{2}\right\| \leq\left\|A\left(I-P_{2^{2 n}}\right) A\right\|+\left\|A P_{2^{2 n}} A-A_{n}^{2}\right\| .
$$

получаем (15). Неравенство (16) доказывается аналогично.

Teорема 4. Пусть $\alpha=\mu_{1} \delta^{1 /(p+1)}, 2^{-2 m} \sqrt{n}=\mu_{2} \delta$ и $B$ - произвольный базис из $\mathcal{B}_{r}$. Для того чтобы $\left(\hat{\Gamma}_{n}, B\right) \in \hat{\Pi}_{\delta, p, p}^{d}\left(\hat{\mathcal{H}}_{\gamma}^{r}\right), p>1$, достаточно выпол̀нения условий

$$
\begin{gathered}
\hat{\chi}_{*} \mu_{1}^{-1}+\rho \hat{\chi}_{p} \mu_{1}^{p}+\rho \hat{\chi}_{0} a_{p / 2}\left(v_{*} \mu_{2}\right)^{\min \{p / 2, l\}}+\rho \hat{\chi}_{*} \mu_{1}^{-1} v_{p} \mu_{2} \leq \\
\leq d_{1} \rho^{1 /(p+1)}, \\
\rho v_{p} \mu_{2} \leq d_{2} .
\end{gathered}
$$

Оптилальная по порядку оченка точности $d_{1} p^{1 /(p+1)} \delta^{p /(p+1)}$ достигается при использовании произвольного $P П М\left(R_{\alpha}, \hat{\Gamma}_{n}, B\right)$, где $R_{\alpha} \in \hat{R}^{\prime}, \quad \hat{p}_{*} \geq p$. $\Pi$ pu этолl $\operatorname{card}\left(\hat{\Gamma}_{n}\right)=2^{2 n}(n+1)=\delta^{-1 / r} \log ^{1+1 /(2 r)}\left(\delta^{-1}\right)$.

Доказательство. Отметим, прежде всего, что в силу (16) при $n$ и $\alpha$, удовлетворяюших условиям теоремы, выполняется $\left\|A-A_{n}\right\| \leq \gamma_{0} \alpha$. Далее, в силу (13) и (15) имеем

$$
\left\|A^{p}-\left|A_{n}\right|^{p}\right\| \leq a_{p / 2}\left\|A^{2}-A_{n}^{2}\right\|^{\min \{p / 2,1\}} \leq a_{p / 2}\left(v_{*} 2^{-2 m} n\right)^{\min \{p / 2,1\}} .
$$

Подставляя в (11) найденную оценку, соотношение (14), а также выражения для параметров $\alpha$ и $n$, получаем условие (17). Для получения (18) достаточно в условие (9) подставить (14).

Замечание 2. В случае $0<p \leq 1$ соответствующий результат (получен в 
$[3,4])$ достигается в рамках традиционной проекционной схемы ( $A_{n}=$ $\left.=P_{2^{n}} A P_{2^{n}}\right)$ и содержится в теореме 2 настоящей работы.

4. В заключение покажем, что найденная в теореме 4 оценка минимального числа функционалов (4) является в смысле величины $\widehat{\mathrm{Card}}_{\delta, p, p}$ точной по порядку.

Теорема 5. Пусть выполнены условия (17), (18). Тогда для $р=2,3, \ldots$ справедливы оченки

$$
c_{1} \delta^{-1 / r} \log \left(\delta^{-1}\right) \leq \widehat{\operatorname{Card}}_{\delta, p, p}\left(\hat{\mathcal{H}}_{\gamma}^{r}\right) \leq c_{2} \delta^{-1 / r} \log { }^{1+1 /(2 r)}\left(\delta^{-1}\right) .
$$

Оптильальный порядок объеліа дискретной инфорлации доставляет схельа $(\Omega, B)$, где $\Omega=\hat{\Gamma}_{n}(12)$, а $B-$ произвольный базис из $\mathcal{B}_{k}$.

Доказательство. Верхняя оценка следует из теоремы 4. Для нахождения нижней оценки рассмотрим случай $p=2$. При $p=3,4, \ldots$ рассуждения аналогичны. Для упрощения выкладоқ будем считать $\rho=1$. Итак, зафиксируем произвольный элемент $(\Omega, B)$ из множества $\hat{\Pi}_{\delta, 2,1}^{d}\left(\hat{\mathcal{H}}_{\gamma}^{r}\right)$. Легко видеть, что в силу (9) справедливо $(1,1) \in \Omega$.

Для любого $x \in X$ и $L=1,2, \ldots$ положим

$$
H_{L} x=b_{1}\left(b_{1}+L^{-r} b_{L}, x\right)+L^{-r} b_{L}\left(b_{1}+b_{L}, x\right) \text {. }
$$

Тогда при любом $\gamma_{1} \leq \gamma\left(2 \sqrt{2} \bar{\beta}_{r}\right)^{-1}$ очевидно включение $\gamma_{1} H_{L} \in \hat{\mathcal{H}}_{\gamma}^{r}$. Заметим, что замена конечномерного оператора $\gamma_{1} H_{L}$ оператором $\gamma_{1} H_{L}+H$, где $H$-произвольный элемент класса $\hat{\mathcal{H}}_{\gamma}^{r}$ такой, что $H b_{L}=H b_{1}=0$, в дальнейших рассуждениях ничего не изменит.

Прежде всего оценим величину $M=\min \{i: i \notin \omega\}$, где, как и прежде, $\omega=$ $=\{i:(i, j) \in \Omega\}$. Для этого рассмотрим уравнение $A_{1} x=f_{1}$, решеннем которого является элемент $x_{1}=A_{1}^{2} b_{1}$, где $A_{1}=\gamma_{1} H_{M}$ и $f_{1}=A_{1}^{3} b_{1}$. Учитывая очевидное соотношение $A_{1, \Omega} x=\gamma_{1} b_{1}\left(b_{1}, x\right)$, находим

$$
\begin{gathered}
A_{1} b_{1}=\gamma_{1}\left(b_{1}+M^{-r} b_{M}\right), \quad x_{1}=\gamma_{1}^{2}\left(1+M^{-2 r}\right) b_{1}+\gamma_{1}^{2}\left(M^{-r}+M^{-2 r}\right) b_{M}, \\
A_{1, \Omega} x_{1}=\gamma_{1}^{3}\left(1+M^{-2 r}\right) b_{1}, \quad P_{\Omega} f_{1}=\gamma_{1}^{3}\left(1+2 M^{-2 r}+M^{-3 r}\right) b_{1} .
\end{gathered}
$$

Отсюда следует $\left\|\left(P_{\Omega} A_{1}-A_{1, \Omega}\right) x_{1}\right\|_{X}=\gamma_{1}^{3}\left(M^{-2 r}+M^{-3 r}\right)$. Тогда в силу (9) получаем, что $M$ удовлетворяет условию $M^{-2 r}+M^{-3 r} \leq d_{2} \delta / \gamma_{1}^{3}$.

Положим $\eta=\max \left\{2 d_{2}, 1\right\}$. Рассмотрим еще одно уравнение $A_{2} x=f_{2}$, решением которого является элемент $x=x_{2}=A_{1, \Omega}^{2} b_{1} / \eta=\gamma_{1}^{2} b_{1} / \eta$, где $A_{2}=$ $=A_{1, \Omega}, f_{2}=\gamma_{1}^{3} b_{1} / \eta$. Имеем

$$
\left\|\frac{x_{1}}{\eta}-x_{2}\right\|_{X} \geq \gamma_{1}^{2} \frac{M^{-r}}{\eta}
$$

Поскольку справедливо

$$
\left\|\frac{P_{\Omega} f_{1}}{\eta}-f_{2}\right\|_{X}=\gamma_{1}^{3} \frac{2 M^{-2 r}+M^{-3 r}}{\eta} \leq \delta,
$$

то в случае $f_{2, \delta}=P_{\Omega} f_{1} / \eta$ наборы функционалов (4) для уравнении $A_{1} x_{1} / \eta=$ $=f_{1} / \eta$ и $A_{2} x_{2}=f_{2, \delta}$ совпадают. Следовательно, для любого $R_{\alpha} \in R$ имеет место соотношение 


$$
x_{\text {disc }}\left(R_{\alpha}, \Omega, B, A_{1}, \frac{f_{1}}{\eta}\right)=x_{\text {disc }}\left(R_{\alpha}, \Omega, B, A_{2}, f_{2, \delta}\right):=R_{\alpha}\left(A_{1, \Omega}\right) P_{\Omega} \frac{f_{1}}{\eta} .
$$

Тогда, учитывая (19), (20), в силу произвольности $R_{\alpha}$ и включения $(\Omega, B) \in$ $\in \hat{\Pi}_{\delta, 2,1}^{d}\left(\hat{\mathcal{H}}_{\gamma}^{r}\right)$, получаем

$$
\begin{aligned}
& \gamma_{1}^{2} \frac{M^{-r}}{\eta} \leq\left\|\frac{x_{1}}{\eta}-x_{2}\right\|_{X} \leq \\
& \leq\left\|\frac{x_{1}}{\eta}-R_{\alpha}\left(A_{1, \Omega}\right) P_{\Omega} \frac{f_{1}}{\eta}\right\|_{X}+\left\|x_{2}-R_{\alpha}\left(A_{1, \Omega}\right) P_{\Omega} \frac{f_{1}}{\eta}\right\|_{X} \leq \\
& \leq \sup _{f_{B}:\left\|f_{1}-f_{\delta}\right\| \leq \delta}\left\|x_{1}-R_{\alpha}\left(A_{1, \Omega}\right) P_{\Omega} f_{\delta}\right\|_{X}+ \\
& +\sup _{f_{\delta}:\left\|f_{2}-f_{\delta}\right\|_{X} \leq \delta}\left\|x_{2}-R_{\alpha}\left(A_{1, \Omega}\right) P_{\Omega} f_{\delta}\right\|_{X} \leq \\
& \leq \sup _{x_{1} \in M_{2,1}\left(A_{1}\right) f_{\delta}:\left\|A_{1} x_{1}-f_{\delta}\right\|_{X} \leq \delta}\left\|x_{1}-x_{\text {disc }}\left(R_{\alpha}, \Omega, B, A_{1}, f_{\delta}\right)\right\|_{X}+ \\
& +\sup _{x_{2} \in M_{2,1}\left(A_{2}\right) f_{\delta}:\left\|A_{2} x_{2}-f_{\delta}\right\|_{X} \leq \delta}\left\|x_{2}-x_{\text {disc }}\left(R_{\alpha}, \Omega, B, A_{2}, f_{\delta}\right)\right\|_{X} \leq \\
& \leq 2 \sup _{A \in \hat{\mathcal{H}}_{\gamma}^{r}} \sup _{x_{0} \in M_{2,1}(A) f_{8}:\left\|A x_{0}-f_{\delta}\right\|_{X} \leq \delta}\left\|x_{0}-x_{\text {disc }}\left(R_{\alpha}, \Omega, B, A, f_{\delta}\right)\right\|_{X} \leq \\
& \leq 2 \mathscr{E}_{\delta, 2,1}\left(\hat{\mathcal{H}}_{\gamma}^{r}, R_{\alpha}, \Omega, B\right) \leq 2 d_{1} \delta^{2 / 3} .
\end{aligned}
$$

Отсюда при $\eta_{1}=\left(\gamma_{1}^{2} /\left(2 d_{1} \eta\right)\right)^{1 / r}$ следует оценка $M \geq M_{1}+1:=\eta_{1} \delta^{-2 /(3 r)}$, где $\left[1, \dot{M}_{1}\right] \subset \omega$.

Далее, на следующих примерах определим точки, принадлежность которых множеству $\Omega$ необходима для включения $(\Omega, B) \subset \hat{\Pi}_{\delta, 2,1}^{d}\left(\hat{\mathcal{H}}_{\gamma}^{r}\right)$.

1. Докажем, что при любых $L, 1 \leq L \leq M_{1}$, все точки $(L, 1),(1, L)$ принадлежат $\Omega$. Предположим противное, а именно, пусть наңдется такое значенне $L, 1<L \leq M_{1}$, что $(L, 1),(1, L) \notin \Omega$ (напомним, что мы рассматриваем область $\Omega$, симметричную относительно диагонали координатной плоскости). Рассмотрим уравнение $A_{3} x=f_{3}$, имеющее решение $x_{3}=A_{3}^{2} b_{1}$, где $A_{3}=$ $=\gamma_{1} H_{L}$ и $f_{3}=\left(\gamma_{\mathrm{i}} H_{L}\right)^{3} b_{1}$. Нетрудно видеть, что

$$
\begin{gathered}
x_{3}=\gamma_{1}^{2}\left(1+L^{-2 r}\right) b_{1}+\gamma_{1}^{2}\left(L^{-r}+L^{-2 r}\right) b_{L}, \\
P_{\Omega} A_{3} x_{3}=f_{3}=\gamma_{1}^{3}\left(1+2 L^{-2 r}+L^{-3 r}\right) b_{1}+\gamma_{1}^{3}\left(L^{-r}+L^{-2 r}+2 L^{-3 r}\right) b_{L} .
\end{gathered}
$$

В то же время элемент $A_{3, \Omega} x_{3}$ может иметь два вида:

$$
A_{3, \Omega} x_{3}=\gamma_{1}^{3}\left(1+L^{-2 r}\right) b_{1}, \text { если }(L, L) \notin \Omega,
$$

и

$$
A_{3, \Omega} x_{3}=\gamma_{1}^{3}\left(1+L^{-2 r}\right) b_{1}+\gamma_{1}^{3}\left(L^{-2 r}+L^{-3 r}\right) b_{L}, \quad \text { если } \quad(L, L) \in \Omega .
$$

В обонх случаях справедливо $\left\|\left(P_{\Omega} A_{3}-A_{3, \Omega}\right) x_{3}\right\|_{X} \geq \gamma_{1}^{3} L^{-r}>\gamma_{1}^{3} M_{1}^{-r} \approx \delta^{2 / 3}$, что при достаточно малых $\delta$ противоречит (9). 
2. Докажем теперь, что если $1<L \leq \eta_{2} \delta^{-1 /(2 r)}$, где $\eta_{2}=\left(\gamma_{1}^{3} / d_{2}\right)^{1 /(2 r)}$, To

$$
(L, L) \in \Omega \text {. }
$$

Как и выше, доказательство проведем от противного. Воспользуемся для этого уравнением (20) из предыдущего примера. А именно, в случае $(L, L) \notin \Omega$ имеем $\left(P_{\Omega} A_{3}-A_{3, \Omega}\right) x_{3}=\gamma_{1} L^{-r} b_{L}\left(b_{L}, x_{3}\right)=\gamma_{1}^{3}\left(L^{-2 r}+L^{-3 r}\right) b_{L}$. Отсюда следует $\left\|\left(P_{\Omega} A_{3}-A_{3, \Omega}\right) x_{3}\right\|_{X}>\gamma_{1}^{3} L^{-2 r} \geq \gamma_{1}^{3} \eta_{2}^{-2 r} \delta=d_{2} \delta$, что в силу (9) доказывает (21).

3. Рассмотрим оператор $A_{4}$ из $\hat{\mathcal{H}}_{\gamma}^{r}$ следующего вида:

$$
\begin{gathered}
A_{4} x=\gamma_{2} b_{1}\left(b_{1}+L^{-r} b_{L}+J^{-r} b_{J}, x\right)+ \\
+\gamma_{2} b_{L}\left(L^{-r} b_{1}+L^{-r} b_{L}+J^{-r} b_{J}, x\right)+\gamma_{2} J^{-r} b_{J}\left(b_{1}+b_{L}+b_{J}, x\right),
\end{gathered}
$$

где $\gamma_{2} \leq \gamma\left(3 \sqrt{3} \bar{\beta}_{r}\right)^{-1}$, а $L$ и $J-$ произвольные целые числа такие, что $1<$ $<L<J \leq M_{1}$ и $L J \leq \eta_{3} \delta^{-1 / r}, \eta_{3}=\left(\gamma_{2}^{3} / d_{2}\right)^{1 / r}$. Из предыдущих примеров следует, что $(1, L),(L, 1)(1, J),(J, 1),(L, L) \in \Omega$. Случаи $(J, J) \notin \Omega$ возможен лишь при $J>\eta_{2} \delta^{-1 /(2 r)}$. Докажем, что $(L, J),(J, L) \in \Omega$. Предположим противное и рассмотрим уравнение $A_{4} x=f_{4}$, решение которого есть $x_{4}=$ $=A_{4}^{2} b_{1}$. Вычислим

$$
\begin{aligned}
\dot{x}_{4}=\gamma_{2}^{2}(1+ & \left.L^{-2 r}+J^{-2 r}\right) b_{1}+\gamma_{2}^{2}\left(L^{-r}+L^{-2 r}+J^{-2 r}\right) b_{L}+ \\
& +\gamma_{2}^{2}\left(J^{-r}+L^{-r} J^{-r}+J^{-2 r}\right) b_{J} .
\end{aligned}
$$

Далее находим (соответственно при $(J, L) \in \Omega$ и в противном случае)

$$
\begin{gathered}
\left(b_{J},\left(P_{\Omega} A_{4}-A_{4, \Omega}\right) x_{4}\right)=\gamma_{2}^{3} J^{-r}\left(L^{-r}+L^{-2 r}+J^{-2 r}\right), \\
\left(b_{J},\left(P_{\Omega} A_{4}-A_{4, \Omega}\right) x_{4}\right)=\gamma_{2}^{3} J^{-r}\left(L^{-r}+J^{-r}+L^{-r} J^{-r}+L^{-2 r}+2 J^{-2 r}\right) .
\end{gathered}
$$

В обоих случаях справедливо $\left\|\left(P_{\Omega} A_{4}-A_{4, \Omega}\right) x_{4}\right\|_{X}>\gamma_{2}^{3}(L J)^{-r} \geq \gamma_{2}^{3} \eta_{3}^{-r} \delta=$ $=d_{2} \delta$, что противоречит включению $(\Omega, B) \in \hat{\Pi}_{\delta, 2,1}^{d}\left(\hat{\mathcal{H}}_{\gamma}^{r}\right)$.

Таким образом, на основании рассмотренных примеров можно сделать вывод:

для того чтобы $(\Omega, B) \in \hat{\Pi}_{\delta, 2.1}^{d}\left(\hat{\mathcal{H}}_{\gamma}^{r}\right)$, необходимо, чтобы все точки $(L, J)$, $1 \leq L, J \leq M_{1}=\eta_{1} \delta^{-2 /(3 r)}-1, L J \leq\left(\eta_{3} / \delta\right)^{1 / r}$, входили в множество $\Omega$.

Осталось подсчитать общее число $N$ таких точек:

$$
N \times d_{2} \delta^{-1 / r} \int_{1}^{M_{1}} \frac{d x}{x}=\delta^{-1 / r} \log \left(\delta^{-1}\right) .
$$

Тем самым теорема полностью доказана.

Аналогично теореме 5 устанавливается следующее утверждение.

Теорема 6. Для достаточно льалых $\delta$ выполняется $\widehat{\operatorname{Card}}_{\delta, 1, p}\left(\hat{\mathcal{H}}_{\gamma}^{r}\right) \times$ $\approx \delta^{-1 / r}$. Оптильальный по порядку объель инфорльации (4)достигается в ральках галеркинской схемы дискретизации при $\Omega=\left[1,2^{n}\right] \times\left[1,2^{n}\right], 2^{n}=$ $=\delta^{-1 /(2 r)}$. 
Замечание 3. Сравнение теорем 2, 5, 6 позволяет сделать следующее заключение. Оказывается, что при дискретизации уравнения (2) замена самосопряженного оператора $A$ на самосопряженный конечномерный оператор $A_{\Omega}$ оправдана лишь в случае $p \leq 1$ (например, в рамках стандартного метода Лаврентьева). В то же время при более высоких значениях $p$,вполне естественная" симметризация дискретной схемы приводит к увеличению объема используемой информации (4).

Замечание 4. Нетрудно видеть, что все приведенные выше результаты справедливы и в случае, когда вместо точного оператора $A$ задано некоторое его приближение $A_{h} \in \mathcal{L}\left(X, X^{r}\right)$ такое, что $\left\|A-A_{h}\right\| \leq h$, где $h \leq c \delta$.

1. Трауб Дж., Вожыцяковский Х. Общая теория оптималыных алюоритмоњ. - М.: Мир, 1983. $382 \mathrm{c}$.

2. Переверзев С. В. Оптимизация метоцов приближенюо о решения операториых уравиений. Киев: ЙI-т математики НАН Укранाны, 1996. - 252 c.

3. Plato R., Vainikko G. On the regularization of the Ritz-Galerkin method for solving III-posed problems // Учен. зan. Tapr. yil-ra. - 1989. - Bisn. 863. - C. 3-17.

4. Plato R., Vainikko $G$. On the regularization of projection methods for solving III-posed problems /I Numer. Math. - 1990. - 57. - P. 63-70.

5. Pereverzev S. V. Optimization of projection methods for solving III-posed problems // Computing. - 1995. - 55. - P. $113-124$.

6. Солодкий С. Г. О цискретизацин пекорректшых задач // Журі. выцислит. математики и мат. физики. - 1996: - 36, № 8. - С. 15-22.

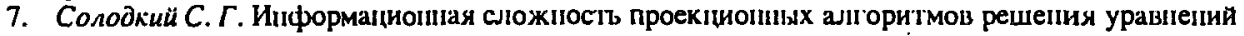
Фредгольма I рода. I // Укр. мaт. журі. - 1998. - 50, № 5. - С. 699-711.

8. Иванов B. K., Васии В. В., Тапала B. П. Теория линейых пекорректных задач и ее приложелияя. - М.: Наука, 1978. - $206 \mathrm{c}$.

9. Тихопо А. Н., Арселии В. Я. Метоцы решения пекорректиљх задач. - М.: Науха. 1979. $285 \mathrm{c}$.

10. Вайликко Г. М., Веретелииков А. Ю. Итерациопиые процелуры в пекоррек т7плх задачах. M.: Hayxa, 1986. - 182 c.

11. Гопчарский А. В., Леопов А. С., Яеола А.Г. Копечпоразпостиая аппроксимация липейних пекорректиых зацач // Журн. вычислит. математики и мат. физики. - 1974. - 14, № 1. - С. 15-24.

12. Dahmen W., Kunoth A., Schneider R. Operator equations, multiscale concepts and complexity /I Lect. Appl. Math. - 1996. - 32. - P. 225-261.

13. Бабенко К. И. О приближения периодических фупкций мпоги перемешпьх тригонометрическими мпогочлегіами // Докл. АН СССР. - 1960. - 132, № 2. - С. 247-250.

14. Бакушинскиї А. Б. Олип общий прием построеєия регуляризующих алгоригмов цля лииейного пекорректиого уравіения в гильбертоном прострапстве // Жури. вычнслит. математики и мат. физики. $-1967 .-7$, № 3. - С. 672-677.

Получчено 22.12.97, после доработки -05.10 .98$ 